\title{
Propuesta de un programa de gestión para mejorar el manejo de los residuos sólidos en el distrito de San Juan de Miraflores
}

\begin{abstract}
RESUMEN
En este artículo se investiga: ¿Cómo se puede mejorar en el distrito de San Juan de Miraflores (SJM) el manejo de los residuos sólidos con respecto al ambiente, el recojo y el comportamiento de la población? Se propone un programa de gestión de manejo de residuos en SJM que se concentra en los problemas de un trato ambientalmente inadecuado de los desechos, un servicio de recojo insuficiente y una cultura de manejo de los residuos no responsable e insostenible.

Se analizó la situación actual con datos que se levantaron a través de trabajo de campo y otras fuentes como resultado del estudio. Para mejorar estos problemas, se propone la separación y el reciclaje de desechos, la mejora de la ruta, del "factor personal" y del "factor humano" para el servicio de recojo y la creación de una consciencia social que asegure un manejo responsable y sostenible de los desechos domiciliarios.
\end{abstract}

Palabras clave: programa de gestión, residuos sólidos, servicio de recojo, impacto ambiental

Proposal of a management program to IMPROVE THE HANDLING OF SOLID WASTE IN THE district San Juan de Miraflores

\section{ABSTRACT}

One investigates in this paper: How can you improve the management of residential waste in the district of San Juan de Miraflores (SJM) concerning the environment, the waste collection and the behavior of the inhabitants? One proposes a program for the management of residential waste in SJM concentrating on the environmentally inadequate handling of waste, the insufficient waste collection service and an irresponsible and not sustainable handling of solid waste.

In order to improve these points, the current situation is analyzed with data gained by field work and other sources. One suggests to separate and recycle the garbage, to improve the route, the factors "employees" and "human" for a better service of waste collection and the creation of a social consciousness to assure a responsible and sustainable handling of the domestic waste.

Keywords: management program, domestic waste, collection service, environmental impact

\section{INTRODUCCIÓN}

En el año 2015 se generó tanta basura como nunca antes en San Juan de Miraflores (SJM): 79952652 kilogramos de desechos (Perú a, 2015). Esto significa que el departamento de Limpieza Pública de dicho distrito tuvo que recoger, transportar y deshacer 222091 kilogramos de residuos sólidos diariamente (Perú a, 2015). Esta tarea implica un gran esfuerzo organizacional y de gestión que actualmente no se está realizando a un nivel adecuado. Las consecuencias son malos olores, enfermedades y una baja calidad de vida de los habitantes de SJM.

Autores como López (2009) y Benavides (2007) ya han analizado situaciones parecidas anteriormente. Sin embargo, esas investigaciones se enfocan en partes específicas de la problemática de los desechos. En el presente trabajo se ofrece una vista más completa que incluye las tres áreas más importantes: el impacto ambiental, el servicio de recojo y la conducta humana sostenible.

La investigación en este tema tiene un gran valor tanto práctico como teórico. El programa de gestión elaborado ofrece soluciones para una vida más saludable y de mejor calidad. Si la gestión de los desechos es más eficiente y eficaz, habrá menos insectos, ratas, malos olores y gases tóxicos en el distrito, lo que significa menos enfermedades. Además, la investigación facilita la aplicación del conocimiento ganado y del programa de gestión a otros distritos en condiciones similares tanto en Lima como en otras ciudades.

El objetivo general es proponer un programa de gestión de manejo de residuos sólidos residenciales en SJM, con el fin de disminuir el impacto ambiental, mejorar el servicio de recojo y sensibilizar a la población.

Esto se detalla en tres objetivos específicos:

O1. Evaluar los impactos ambientales significativos del manejo de los residuos sólidos y sugerir acciones para reducir el impacto ambiental.

O2. Analizar el servicio de recojo, determinar sus factores influyentes y proponer acciones para mejorar el servicio.

* Bachelor of Science. Jefa de Ventas de la agencia Inkaland Tours. E-mail: f.oldenhage@gmail.com 
O3. Analizar los problemas presentes con respecto a la conducta de la población y elaborar un plan de acción para sensibilizar a la población para el manejo responsable y sostenible de desechos.

Revisando el estado de arte, se encontró algunas investigaciones con la misma situación problemática, pero con enfoques diferentes. Lopez Rivera (2009) por ejemplo propuso un programa para el manejo de residuos sólidos en la plaza de un mercado mejorando las prácticas de separación de desechos y las deficiencias en el almacenamiento de los residuos para cumplir con las normas legales y evitar impactos ambientales negativos. Y Benavides (2007) modeló una gestión integral de rellenos sanitarios manuales en Ecuador mejorando la coordinación del servicio de recojo y tomando en cuenta los impactos ambientales.

\section{SOLUCIÓN PROPUESTA}

\subsection{Información básica de SJM y el ciclo de vida de los residuos sólidos}

El 12.01.1965 se fundó el distrito de SJM que se divide en seis zonas, una de ellas es la Zona Urbana donde se realizó la investigación. En total, SJM tiene una superficie de 23,98 kilómetros cuadrados (Perú b, 2015) y una población de aproximadamente 529710 personas para año 2016 (Perú a, 2015). El porcentaje de la población que no tiene una educación que supera la primaria es de $31 \%$ (Perú a, 2015).

En la Figura 1 se puede observar el resumen del ciclo de vida de los desechos en SJM. Este se divide en tres pasos: La generación y preparación de desechos que realizan personas naturales, es decir los habitantes de SJM.

1) El recojo de los desechos que es realizado por la Municipalidad de SJM.

2) La deposición que también es realizado por la Municipalidad de SJM.

El programa de gestión propuesto es dividido según este ciclo de vida en las problemáticas del impacto ambiental que ocurre principalmente en la deposición final y en la generación, el servicio de recojo que corresponde al segundo paso de ciclo de vida de los desechos y la cultura de manejo de los desechos que mejora los problemas encontrados en el primer paso de la generación y preparación de los residuos sólidos.
Figura 1. Ciclo de vida de residuos sólidos domiciliarios en SJM.

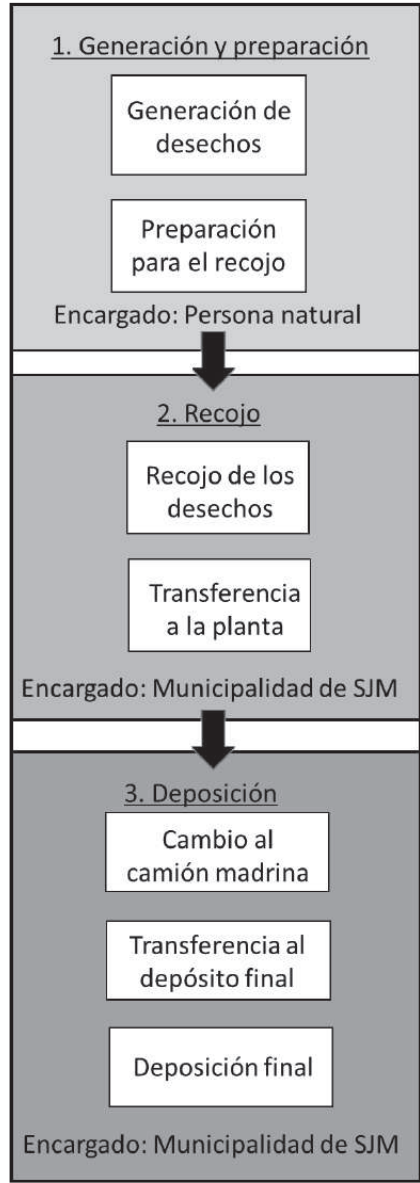

Fuente: Elaboración propia.

\subsection{Impacto ambiental}

Con $97,52 \%$ en el año 2004, la gran mayoría de los residuos sólidos en SJM es generada en los domicilios (Perú a, 2004).

Según el artículo N. ${ }^{\circ}$ 119.1. "Del manejo de los residuos sólidos" de la Ley General del Ambiente - Ley N. ${ }^{\circ} 28611$ del año 2005, la gestión de los residuos sólidos domésticos es responsabilidad de los gobiernos locales, es decir de las municipalidades de cada distrito. En la segunda parte del mismo artículo se detalla que la responsabilidad de la municipalidad es hasta la deposición final de los desechos siempre considerando los criterios de la minimización de impactos sociales y ambientales negativos. Por eso, la municipalidad de SJM tiene que hacerse cargo de los problemas ambientales.

Uno de los mayores problemas encontrados en el distrito es que el gobierno local no tiene el nivel técnico ni administrativo para una gestión adecuada (Perú b, 2004). Además, a pesar de que SJM tiene su propia planta de transferencia, no puede 
usarla por falta de una licencia y los permisos correspondientes. Actualmente, la municipalidad de SJM contrata los servicios de la empresa Servicios Generales Rambell para transportar los desechos al relleno sanitario de Portillo Grande que causa mayores gastos de lo necesario (Perú a, 2015). Otro problema es que se recoge todos los residuos juntos sin separar los diferentes tipos de desechos que no permite reciclar. Los desechos tampoco reciben ningún tipo de tratamiento en el depósito final. Se trata simplemente de confinar los residuos en la menor área posible para reducir el volumen (Perú a, 2015). Otro problema es que la misma población tiene una limitada cultura ambiental y poco conocimiento sobre el trato sostenible de desechos (Perú b, 2004). Además, la gestión deficiente de los residuos sólidos actual tampoco cumple con las leyes y normas establecidas.
Con la matriz de Leopold, que se observa en la Tabla 1, se califica los impactos ambientales como severos y dañinos tanto al corto como al largo plazo. Para poder enfrentar los problemas descubiertos, se propone cinco proyectos de mejora:

1) Capacitación del personal administrativo

2) Separación de residuos sólidos domésticos

3) Compostaje de los desechos orgánicos

4) Mejoras en el transporte y deposición final de los desechos inorgánicos

5) Capacitación de la población

Cada uno de estos proyectos se detalla especificando los fines/impactos, el efecto, los componentes, las actividades, los costos, las personas involucradas y el plazo para facilitar la implementación.

Tabla 1. Matriz de Leopold

\begin{tabular}{|c|c|c|c|c|c|c|c|c|c|c|c|c|c|c|}
\hline \multirow{3}{*}{ 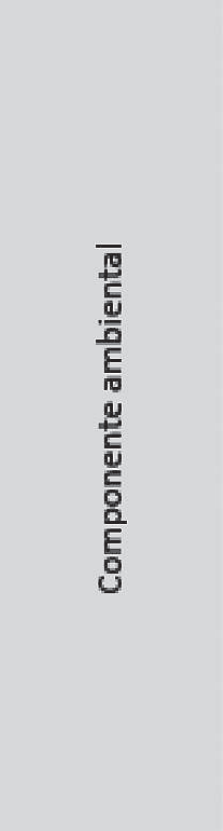 } & \multicolumn{2}{|c|}{ Indicador } & \multicolumn{8}{|c|}{ Factores de residuos sólidos } & & & & \\
\hline & \multirow{2}{*}{ 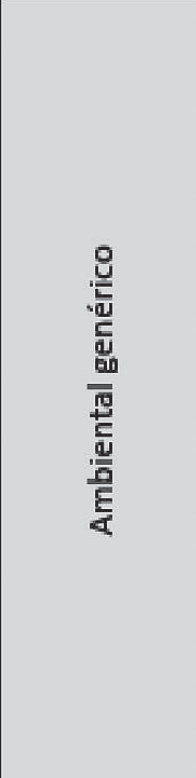 } & \multirow{2}{*}{ 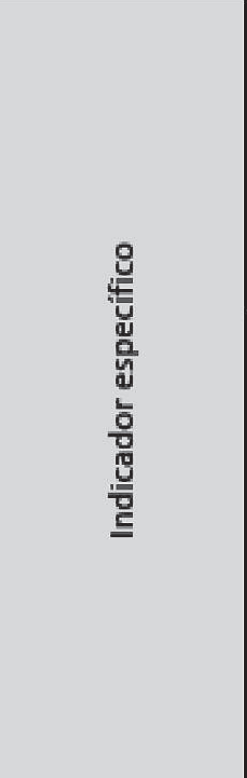 } & \multicolumn{2}{|c|}{ 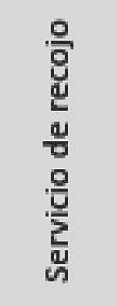 } & \multicolumn{2}{|c|}{ 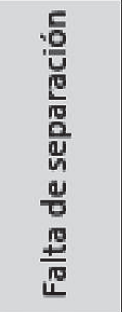 } & \multicolumn{2}{|c|}{ 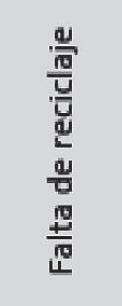 } & \multicolumn{2}{|c|}{ 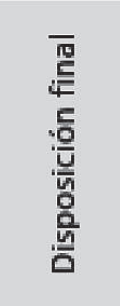 } & & & & \\
\hline & & & 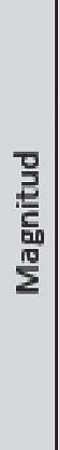 & 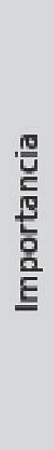 & 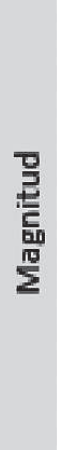 & 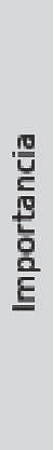 & 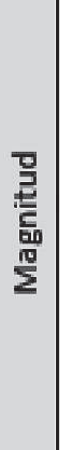 & 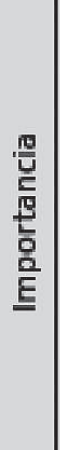 & 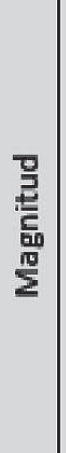 & 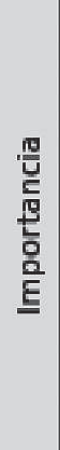 & 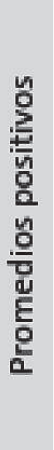 & 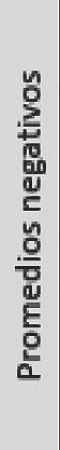 & 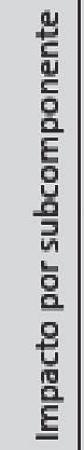 & 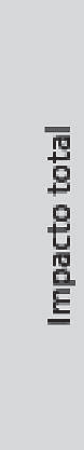 \\
\hline Atmosférico & $\begin{array}{l}\text { Calidad del } \\
\text { Aire }\end{array}$ & $\begin{array}{l}\text { Emisiones de } \\
\text { malos olores }\end{array}$ & -3 & 3 & -3 & 2 & & & & & & 2 & -15 & \\
\hline Hidrosférico & Calidad & Agua & & & -3 & 3 & & & -3 & 5 & & 2 & -24 & \\
\hline Paisajístico & $\begin{array}{l}\text { Calidad } \\
\text { visual }\end{array}$ & Calidad paisaje & -2 & 2 & -4 & 3 & & & & & & 2 & -16 & \\
\hline \multirow[t]{2}{*}{$\begin{array}{l}\text { Antropos- } \\
\text { férico }\end{array}$} & $\begin{array}{l}\text { Calidad de } \\
\text { vida }\end{array}$ & $\begin{array}{l}\text { Felicidad en el } \\
\text { vecindario }\end{array}$ & -3 & 2 & 2 & 2 & & & & & 1 & 1 & -2 & -122 \\
\hline & Salud & Enfermedades & -4 & 5 & & & & & & & & 1 & -20 & \\
\hline Sostenibilidad & Desperdicio & $\begin{array}{l}\text { Cantidad } \\
\text { recursos } \\
\text { naturales }\end{array}$ & & & & & -5 & 4 & -5 & 5 & & 2 & -45 & \\
\hline
\end{tabular}

Fuente: Elaboración propia. 


\subsection{Servicio de recojo}

Para entender mejor el proceso de recojo de los desechos sólidos que se está usando en el distrito, se visualiza en un mapa de proceso. Después, se hace un Análisis de Modal de Efectos y Fallos (AMEF) para identificar los problemas y priorizarlos. Las actividades más críticas que se encuentran son las siguientes:

1) Los ayudantes recogen las bolsas; los desechos se dispersan en el suelo y los ayudantes se enferman por el contacto directo con los desechos.

2) Viaje al depósito/vertedero; tomar una ruta no óptima implica demoras en el servicio que aumentan los costos.

3) Asignar el chofer a un camión y ruta; ya que no hay un equipo fijo, hay demoras y una mayor probabilidad de daños.

4) El chofer maneja el camión por las calles; carros mal estacionados, rejas cerradas y pistas en mal estado causan demoras que aumentan los costos.

Con el Ishikawa, que se puede observar en la Figura 2, se identifica tres causas raíces de estos problemas en base a su magnitud, frecuencia e impacto en el proceso:
1) Ruta inadecuada

2) Malas condiciones laborales

3) Inconsciencia de la población

Para poder comprobar si los tres factores encontrados anteriormente influyen en la efectividad del servicio de recojo, primero hay que elaborar los datos claves para el proceso de análisis. Se tomó dos muestras en la zona A de la Zona Urbana de SJM, la primera en marzo de 2015 y la segunda en septiembre de 2015. El resultado de este trabajo de campo se presenta en la Tabla 2. La ruta 1 es la ruta encontrada en marzo de 2015 y la ruta 2 corresponde a la ruta usada en septiembre de 2015. El factor humano se mide de 1 a 3 considerando 3 como el grado de mayor influencia negativa humana. En el caso del factor personal también se mide de 1 a 3 pero esta vez 1 representa el valor más negativo con un personal muy poco capacitado. Los costos se refieren a los costos directos del servicio de recojo en soles. Una alta calidad de servicio es representada por el valor 1 que significa que las calles están limpias después de que pasó el camión de recojo. La relación entre los costos y la calidad de servicio obtenido se refleja en la eficiencia negativa. El servicio con la eficiencia negativa más baja es el mejor.

Analizando estos datos con el programa Minitab 17 , se resuelve que los tres factores influyen

Figura 2. Ishikawa del servicio de recojo.

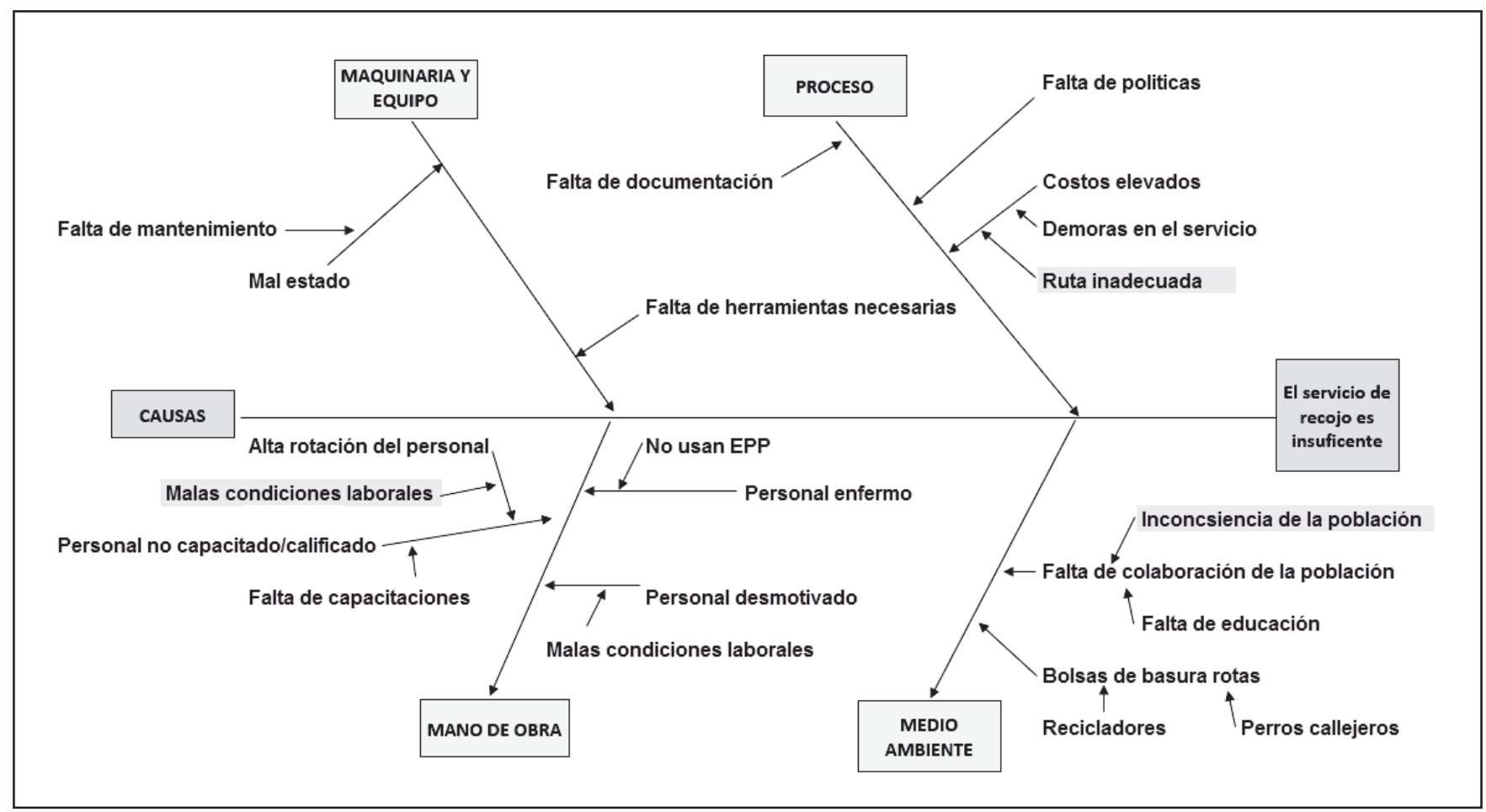

Fuente. Elaboración propia. 
Tabla 2. Datos claves para el proceso de análisis de recojo

\begin{tabular}{|c|c|c|c|c|c|c|}
\hline Día & Ruta & $\begin{array}{c}\text { Factor } \\
\text { humano }\end{array}$ & Personal & Costos & $\begin{array}{c}\text { Calidad de } \\
\text { servicio }\end{array}$ & $\begin{array}{c}\text { Eficiencia } \\
\text { negativa }\end{array}$ \\
\hline 1 & 1 & 2 & 1 & 84.66 & 3 & 110.06 \\
\hline 2 & 1 & 3 & 1 & 86.93 & 3 & 113.01 \\
\hline 3 & 1 & 3 & 1 & 80.87 & 2 & 97.05 \\
\hline 4 & 1 & 1 & 2 & 73.00 & 2 & 87.60 \\
\hline 5 & 1 & 1 & 3 & 81.63 & 1 & 89.79 \\
\hline 6 & 1 & 3 & 3 & 83.90 & 1 & 92.29 \\
\hline 7 & 1 & 3 & 2 & 82.39 & 3 & 107.10 \\
\hline 8 & 2 & 2 & 1 & 71.42 & 1 & 78.56 \\
\hline 9 & 2 & 3 & 1 & 80.20 & 2 & 96.24 \\
\hline 10 & 2 & 1 & 1 & 72.03 & 2 & 86.43 \\
\hline 11 & 2 & 1 & 3 & 56.43 & 1 & 62.07 \\
\hline 12 & 2 & 2 & 2 & 70.81 & 1 & 77.90 \\
\hline 13 & 2 & 1 & 3 & 66.73 & 2 & 80.07 \\
\hline 14 & 2 & 1 & 3 & 66.88 & 1 & 73.57 \\
\hline
\end{tabular}

Fuente. Elaboración propia.

significativamente en la eficiencia del servicio de recojo ya que en los análisis Anova resultaron los R2-ajustados de la ruta de $48,59 \%$, del factor humano de $34,49 \%$ y del personal de $17,27 \%$. La suma de los tres R2-ajustados es muy cerca de $100 \%$ que significa que estos tres factores son los únicos que influyen en el servicio de recojo.

Para mejorar el servicio de recojo, se elabora una nueva ruta con la experiencia ganada en el trabajo de campo. En comparación con las rutas usadas en marzo y septiembre de 2015 , la ruta propuesta reduce la distancia recorrida en un $14 \%$ y $12 \%$ respectivamente. En el caso del factor humano, se propone diferentes métodos de mejora, entre los cuales el sistema de semáforo es el más usado. La idea es indicar que comportamiento disminuye la eficiencia del servicio de recojo en tres pasos según los colores de un semáforo y tomar acciones correctivas en el caso de una cuarta falta. El plan de mejora para el personal se basa principalmente en capacitaciones y mejoras de las condiciones laborales de los empleados para conseguir mejores resultados.

Suponiendo que se implementa las mejoras propuestas, se calcula con la ayuda del Minitab 17 que las medidas propuestas probablemente mejoran la eficiencia del servicio de recojo en un $23,60 \%$, como se puede apreciar en la Figura 3.

Figura 3. Resultado del análisis Anova de la ruta mejorada en Minitab 17.

\begin{tabular}{|c|c|c|}
\hline \multicolumn{3}{|c|}{$\begin{array}{l}\text { ANOVA de un solo factor: Eficiencia negativa mejorada vs. Ruta mejorada } \\
\text { Resumen del modelo }\end{array}$} \\
\hline $\begin{array}{rr}S & \text { R-cuad. } \\
9.01586 & 29.48 \%\end{array}$ & $\begin{array}{r}\text { R-cuad. } \\
\text { (ajustado) } \\
23.60 \%\end{array}$ & $\begin{array}{l}\text { R-cuad. } \\
\text { (pred) } \\
4.02 \%\end{array}$ \\
\hline
\end{tabular}

Fuente. Elaboración propia. 


\subsection{Manejo responsable y sostenible de desechos}

Para que las mejoras propuestas del impacto ambiental y del servicio de recojo tengan éxito, se necesita el apoyo y la buena voluntad de la población. Los problemas más significativos encontrados en este sector son la tendencia creciente de una mayor generación de desechos por persona, la falta de separación de los residuos, el almacenamiento inadecuado de los desechos y la generación de demoras en el servicio de recojo.

Para mejorar esta situación, se elabora un plan de acción con los siguientes seis programas y sus métodos respectivos:

1) Disminución de la cantidad de basura; campañas en las calles y en los colegios explicando el principio de minimización y motivación.

2) Separación de desechos; volantes y pizarras informativas.

3) Creación de una conciencia para almacenar la basura adecuadamente; informar y capacitar a la población.

4) Facilitación del recojo; volantes y el sistema semáforo.

5) Mejorar el porcentaje de pagos de arbitrios a un $60 \%$ en 2016; sanciones y amnistías.

6) Calles limpias; colaboración con colegios y empresas.

\subsection{Programa de gestión}

Durante la elaboración de las diferentes propuestas, se encuentra continuamente relaciones entre los programas propuestos. Por eso, se debe ver las tres categorías del impacto ambiental, del servicio de recojo y del manejo responsable y sostenible de los desechos como un conjunto en el que las propuestas se completan mutuamente. El presupuesto previsto para el programa de gestión es de 2500 000,00 soles incluyendo un margen de seguridad y el plazo planificado para la realización del programa de gestión es de tres años.

\section{CONCLUSIONES}

1. El programa de gestión ofrece propuestas de mejoras para varios problemas del distrito en mención como por ejemplo ahorros por el uso de una planta de transferencia.

2. Se estima que la eficiencia del servicio de recojo mejorará en $23,60 \%$ aplicando las medidas propuestas. Implementando el plan de acción elaborado, los habitantes de SJM estarán más atentos a un trato sostenible de la basura que incluye que ya no se bote basura en la calle.

3. El presupuesto de las actividades y el cronograma muestran que el programa de gestión es fácil de financiar porque solamente implica un pago único adicional por habitante de SJM de 6,53 soles y estará terminado dentro de un mediano plazo.

Para que el éxito del programa de gestión sea mayor, se recomienda en primer lugar las siguientes acciones:

1) A largo plazo se debe hacer una separación más minuciosa de los desechos domésticos para poder reciclar un mayor porcentaje de los residuos como plásticos y papel.

2) A largo plazo se debe optar por camiones que permitan un control del peso de la basura recogida para poder relacionar la cantidad de basura producida con el monto de pago por el servicio de recojo y así crear un incentivo para producir menos desechos.

\section{REFERENCIAS BIBLIOGRÁFICAS}

[1] Benavides, N. A. (2007). Modelo de gestión integral de rellenos sanitarios manuales, para poblaciones entre 15.000 y 30.000 habitantes en el Ecuador (Tesis de posgrado), Universidad Internacional SEK, Quito, Ecuador.

[2] López, N. C. (2009). Propuesta de programa para manejo de residuos sólidos en la plaza del mercado de Cerete, Cerebastos - Córdoba (Tesis de posgrado), Pontificia Universidad Javeriana, Bogotá, Colombia.

[3] Perú a (2004). Plan integral de gestión ambiental de residuos sólidos. Municipalidad de San Juan de Miraflores, Unión Europea, Ministerio de la Mujer y Desarrollo Social. Lima: Autores.

[4] Perú a (2015). Municipalidad Distrital de San Juan de Miraflores, Gerencia de Gestión Ambiental, Sub-Gerencia de Limpieza Pública Plan de manejo de los Residuos Sólidos del Distrito de San Juan de Miraflores - 2015. Lima: Autores.

[5] Perú b (2004). Oficina de asesoría y consultoría ambiental, Instituto de promoción del desarrollo sostenible. Estudio de caracterización física de los residuos sólidos en el distrito de San Juan de Miraflores, PROPOLI. Lima: Autores.

[6] Perú b (2015). Municipalidad distrital de San Juan de Miraflores. Presupuesto institucional de apertura PIA - 2015. Lima: Autores. 